\title{
High cerebral insulin sensitivity is associated with loss of body fat during lifestyle intervention
}

\author{
O. Tschritter • H. Preissl • A. M. Hennige • T. Sartorius • \\ K. T. Stingl • M. Heni $\cdot$ C. Ketterer • N. Stefan • \\ J. Machann • E. Schleicher • A. Fritsche $\cdot$ H.-U. Häring
}

Received: 13 April 2011 / Accepted: 18 August 2011 /Published online: 17 September 2011

(C) Springer-Verlag 2011

\begin{abstract}
Aims/hypothesis Loss of weight and body fat are major targets in lifestyle interventions to prevent diabetes. In the brain, insulin modulates eating behaviour and weight control, resulting in a negative energy balance. This study aimed to test whether cerebral insulin sensitivity facilitates reduction of body weight and body fat by lifestyle intervention in humans.

Methods The study was performed as an additional arm of the TUebingen Lifestyle Intervention Program (TULIP). In 28 non-diabetic individuals (14 female/14 male; mean \pm SE age $42 \pm 2$ years; mean \pm SE BMI $29.9 \pm 0.8 \mathrm{~kg} / \mathrm{m}^{2}$ ), we measured cerebrocortical insulin sensitivity by using magnetoencephalography before lifestyle intervention. Total and visceral fat were measured by using MRI at baseline and after 9 months and 2 years of lifestyle intervention.
\end{abstract}

O. Tschritter $(\bowtie) \cdot$ A. M. Hennige $\cdot$ T. Sartorius $\cdot$ M. Heni $\cdot$

C. Ketterer $\cdot$ N. Stefan $\cdot$ E. Schleicher $\cdot$ A. Fritsche $\cdot$ H.-U. Häring

Department of Internal Medicine IV, University Hospital,

University of Tübingen,

Otfried-Müller-Str. 10,

72076 Tübingen, Germany

e-mail: otto.tschritter@med.uni-tuebingen.de

H. Preissl $\cdot$ K. T. Stingl

Institute of Medical Psychology and Behavioral Neurobiology,

University of Tübingen,

Tübingen, Germany

H. Preissl

Department of Obstetrics and Gynecology, College of Medicine, University of Arkansas for Medical Sciences,

Little Rock, AR, USA

J. Machann

Section on Experimental Radiology, Department of Diagnostic

Radiology, University of Tübingen,

Tübingen, Germany
Results Insulin-stimulated cerebrocortical theta activity at baseline correlated with a reduction in total adipose tissue $(r=-0.59, p=0.014)$ and visceral adipose tissue $(r=-0.76$, $p=0.001)$ after 9 months of lifestyle intervention, accompanied by a statistical trend for reduction in body weight change ( $r=-0.37, p=0.069)$. Similar results were obtained after 2 years.

Conclusions/interpretation Our results suggest that high insulin sensitivity of the human brain facilitates loss of body weight and body fat during lifestyle intervention.

Keywords Body fat · Brain · Insulin sensitivity

$\begin{array}{ll}\text { Abbreviations } \\ \text { IAT } & \text { Individual anaerobic threshold } \\ \text { IHL } & \text { Intrahepatic lipids } \\ \text { MEG } & \text { Magnetoencephalography } \\ \text { MRS } & \text { Magnetic resonance spectroscopy } \\ \text { TAT } & \text { Total adipose tissue } \\ \text { VAT } & \text { Visceral adipose tissue }\end{array}$

\section{Introduction}

In developed countries, obesity and type 2 diabetes mellitus have reached epidemic proportions resulting in high healthcare costs. The prevalence of both conditions is still growing. In recent years, lifestyle interventions have been developed to prevent diabetes $[1,2]$. These interventions usually aim at a moderate weight loss ( $\sim 5 \%$ of initial body weight), reduction in fat intake ( $<30 \%$ of energy), low intake of saturated fatty acids ( $<10 \%$ of energy), increase in physical activity ( $>3 \mathrm{~h}$ per week) and increase in fibre intake (>15 g per 4,187 kJ [1,000 kcal]). Although the mean loss in body weight observed during lifestyle 
intervention programmes is relatively small ( $\sim 3 \%$ of initial body weight), a more pronounced loss of visceral fat (12\%) and liver fat $(33 \%)$ and improvement in peripheral insulin sensitivity have been observed, and these effects are considered to mediate diabetes-preventing effects [3]. However, in a considerable number of participants, these lifestyle interventions seem to be ineffective, and, in particular, body weight change is very heterogeneous. The cerebral insulin signal represents a feedback loop on body weight regulation and mediates weight loss, reduction in food intake and increased energy expenditure [4]. Measurement of cerebrocortical activity by magnetoencephalography (MEG) has revealed that, in obese humans, the brain is resistant to insulin [5]. Although cerebral insulin sensitivity and peripheral insulin sensitivity show a significant positive correlation [5], cerebral insulin sensitivity does not depend on common circulating factors controlling peripheral sensitivity, except for NEFAs [6]. It is unclear whether high insulin sensitivity of the brain may ameliorate loss of weight and body fat during lifestyle intervention.

To address this question, we recruited volunteers for measurement of cerebrocortical insulin effects before participation in a lifestyle intervention (TUebingen Lifestyle Intervention Program [TULIP] study).

\section{Methods}

Participants and experimental design We studied 28 human individuals who were healthy by self-report and clinical investigation and found to be non-diabetic in an OGTT according to ADA criteria. The characteristics of the participants are shown in Table 1. After the OGTT, all volunteers participated in a two-step hyperinsulinaemiceuglycaemic clamp and a saline experiment with repetitive measurement of cerebrocortical activity by MEG. Both experiments were carried out in a blinded, randomised

Table 1 Participants' characteristics

\begin{tabular}{ll}
\hline Characteristic & Value \\
\hline$N$ & 28 \\
Sex (male/female) & $14 / 14$ \\
Age (years) & $42 \pm 2$ \\
BMI $\left(\mathrm{kg} / \mathrm{m}^{2}\right)$ & $29.9 \pm 0.8$ \\
Waist/hip ratio & $0.92 \pm 0.02$ \\
Fasting plasma glucose $(\mathrm{mmol} / \mathrm{l})$ & $5.1 \pm 0.1$ \\
120 min plasma glucose $(\mathrm{mmol} / \mathrm{l})^{\mathrm{a}}$ & $6.6 \pm 0.3$ \\
Fasting plasma insulin $(\mathrm{pmol} / \mathrm{l})$ & $68 \pm 7$ \\
120 min plasma insulin $(\mathrm{pmol} / \mathrm{l})^{\mathrm{a}}$ & $541 \pm 92$ \\
\hline
\end{tabular}

Where applicable, values are mean $\pm \mathrm{SE}$

${ }^{\mathrm{a}}$ OGTT crossover design. Total body fat and fat distribution were measured by MRI and magnetic resonance spectroscopy (MRS) in 26 participants. For safety reasons, participants with claustrophobia, metal implants (e.g. after bone surgery) or tattoos that contain metal had to be excluded from the MRI/MRS measurements. After these baseline measurements, all participants started a lifestyle intervention designed to prevent diabetes. After 9 months and 2 years, the participants were re-evaluated, and the OGTT and MRS/MRI were repeated. Although all 28 individuals participated in the follow-up after 9 months, five dropped out before the end of 2 years and therefore were only used for 9-month analyses and baseline data. The study protocol was approved by the ethics committee of the University of Tübingen, and all participants gave written informed consent before the study.

Oral glucose tolerance test After an overnight fast (at least $10 \mathrm{~h}$ ), all individuals underwent a $75 \mathrm{~g}$ OGTT, and venous blood samples were obtained at 0,30,60, 90, and $120 \mathrm{~min}$ for determination of plasma glucose and insulin. Peripheral insulin sensitivity was calculated from glucose and insulin concentrations during the OGTT as proposed by Matsuda and DeFronzo [7].

Measurement of total adipose tissue, visceral adipose tissue and intrahepatic lipid by magnetic resonance examinations A whole body imaging protocol was used to record a set of 90-120 parallel transverse slices. As the details of the imaging protocol have been previously described in detail [8], we only give a shortened description here. T1-weighted contrast was applied allowing semi-automatic quantitative assessment of fatty tissue and other tissue types in each cross-section. This approach enabled quantification of body volume, total adipose tissue (TAT) (as a percentage of body weight) and total mass of specific fat depots such as visceral adipose tissue (VAT). For measurement of VAT (intraperitoneal and retroperitoneal fat), all slices between the diaphragm and the heads of femora were used.

Intrahepatic lipid (IHL) was determined by localised proton MRS (repetition time, $4 \mathrm{~s}$; echo time, $10 \mathrm{~ms} ; 32$ scans) in the seventh segment of the liver. The lipid content was quantitatively assessed by analysing the signal integral (methylene and methyl signals between 0.7 and $1.5 \mathrm{ppm}$ ), using the liver lipid and water signal integral as internal reference.

Hyperinsulinaemic-euglycaemic clamp and saline experiment with measurement of cerebrocortical activity by MEG All volunteers participated in an insulin and a placebo (saline) experiment in random order on 2 different days approximately 1-2 weeks apart. The participants fasted overnight for at least $10 \mathrm{~h}$. Each experiment started at approximately 
07:00 hours and consisted of a $30 \mathrm{~min}$ baseline period and a two-step hyperinsulinaemic-euglycaemic clamp or saline infusion. Human insulin was applied as a bolus-primed continuous infusion. In the first step, the bolus dose was $6.25 \mathrm{mU} / \mathrm{kg}$, and the infusion dose was $0.25 \mathrm{mU} \mathrm{kg}^{-1} \mathrm{~min}^{-1}$. In the second step, these doses were $17.75 \mathrm{mU} / \mathrm{kg}$ and $1.0 \mathrm{mU} \mathrm{kg}{ }^{-1} \mathrm{~min}^{-1}$, respectively. To maintain blood glucose at baseline levels, a standard hyperinsulinaemiceuglycaemic clamp protocol was followed. Blood glucose was measured at the bedside every 5 to $10 \mathrm{~min}$, and the infusion rate of exogenous glucose was adjusted appropriately to maintain euglycaemia. The details of the clamp procedures have been described by Tschritter et al. [5] During the baseline period and at the end of each experimental step, cerebrocortical activity was measured by MEG. In every individual, a saline experiment was performed to control for non-insulin-derived effects and diurnal variation of cerebrocortical activity.

Magnetoencephalography For each individual the magnetic activity was calculated in accordance with standard procedures. Magnetoencephalographic signals were recorded with a 151 channel whole-head MEG system. The participants were seated in a chair in a magnetically shielded room, and the head was positioned as tight as possible to the MEG sensors. In addition, the position of the head in relation to the sensors was determined by three head coils, attached to the participant's nasion and left and right periauricular points, at the beginning and end of each recording session. If the head movement exceeded $0.5 \mathrm{~cm}$ between start and end of the recording session, the measurement was repeated.

Data were recorded in a continuous mode with a $625 \mathrm{~Hz}$ sampling rate. The recording started with measurement of spontaneous cortical activity during eyes open and closed periods (counterbalanced over sessions and participants) for $1.5 \mathrm{~min}$ each. Thereafter a standard frequency auditory mismatch experiment was applied (data not shown). For each participant the power spectrum for the spontaneous activity was analysed by a standard statistical mapping procedure. As measures of the cerebrocortical insulin effect, changes in beta activity and theta activity during the insulin experiment corrected for the placebo experiment were used as previously described [5].

Lifestyle intervention The lifestyle intervention used here was performed according to the TULIP study as described in [9]. After the baseline measurements, individuals underwent nutrition counselling and had up to 10 sessions with a dietitian. During each visit, participants presented a 3-day food diary and discussed the results with the dietitians. Diet composition was determined with a validated computer program using two representative days of a 3-day diary (DGE-PC 3.0; Deutsche Gesellschaft für Ernährung, Bonn, Germany).

At the beginning of the lifestyle intervention, the participants were instructed to reduce their body weight by $\geq 5 \%$ compared with baseline. Later, they were asked to retain this change in body weight throughout the study. Nutrition counselling also aimed to reduce intake of energy from fat to $<30 \%$ of energy consumed and to increase intake of fibre to at least $15 \mathrm{~g}$ per $4,187 \mathrm{~kJ}(1,000 \mathrm{kcal})$. Furthermore, the participants were asked to reduce intake of saturated fat to $\leq 10 \%$. This dietary regimen was adapted from the diet intervention used in the Diabetes Prevention Study [1].

Individuals were asked to perform at least $3 \mathrm{~h}$ of moderate sports activity per week. Endurance exercise (e.g. walking, swimming) was encouraged. All individuals underwent measurement of the individual anaerobic threshold (IAT). This is considered to be the critical value where a further increase in exercise intensity results in a switch from aerobic to anaerobic metabolism. The heart rate at this IAT can be used to monitor this critical point. For approximately 6 months, the participants were given a heart rate monitor (Polar, Büttelborn, Germany) consisting of a waterproof chest transmitter to detect the electrical signal on the skin which was transmitted continuously and wirelessly using an electromagnetic field to the wrist receiver. Individuals were instructed to exercise while wearing this watch. A heart rate above the IAT reference was considered too strenuous, and individuals were asked to exercise with a heart rate below this reference point.

After practising the estimation of the recommended range of exercise strength during the first 6 months, the participants had to continue the exercise without simultaneous control of heart rate. They were asked to protocol their exercise on a form, including information about time point and kind and duration of exercise, and bring the protocols with them to the nutrition counselling.

The dietitian analysed the protocols after the lifestyle intervention and determined whether, on average, the exercise goal (more than $3 \mathrm{~h}$ per week) had been reached by the individual. The dietitian also assessed whether the other targets (reduction of body weight by more than $5 \%$ of initial weight, reduction of fat intake to below $30 \%$ and saturated fat to below $10 \%$ of energy consumed, and increased intake of fibre to at least $15 \mathrm{~g}$ per $4,187 \mathrm{~kJ}$ $[1,000 \mathrm{kcal}])$ had been reached.

Analytical procedures Plasma glucose was determined during the OGTT using the glucose oxidase method (YSI; Yellow Springs Instruments, Yellow Springs, CO, USA). Blood glucose was determined in the clamp experiments by a HemoCue blood glucose photometer using the glucose 
dehydrogenase method (HemoCue AB, Aengelholm, Sweden). Plasma insulin concentrations were determined by microparticle enzyme immunoassay (Abbott Laboratories, Tokyo, Japan).

Statistical analysis For statistical analyses, MEG data from the saline experiment were subtracted from data from the insulin experiment to correct for potential saline-derived changes and daytime variation. The change in the investigated MEG variables from basal to the second step was considered to indicate the total insulin effect and was used in further analyses.

Changes in body weight, body fat, specific fat depots and metabolic variables during lifestyle intervention and their relation to baseline cerebral insulin sensitivity were analysed by multiple regression analyses. The outcome variable (e.g. VAT after lifestyle intervention) was treated as the dependent variable, while the baseline variable (e.g. VAT before lifestyle intervention), insulin sensitivity of the brain and covariates (as needed) were treated as independent variables. All data were adjusted for sex and age by including these variables in the regression model. Furthermore, interaction between sex and cerebral insulin sensitivity was tested. Changes in plasma glucose, TAT, VAT and IHL were also adjusted for the change in body weight.

Unless otherwise stated, data are mean \pm SE. Nonnormally distributed variables (Shapiro-Wilk W test) were logarithmically transformed. To adjust for covariates and to identify independent relationships, we performed linear multiple regression analyses. $p<0.05$ was considered to indicate significance. For all statistical analyses, the software package JMP (SAS Institute, Cary, NC, USA) was used.

\section{Results}

Effects of lifestyle intervention on body weight, TAT, VAT and liver fat (IHL) During the lifestyle intervention, a mean change in body weight of $-2.7 \pm 0.8 \mathrm{~kg}$ was achieved after 9 months ( $p=0.002)$, and $-1.5 \pm 1.2 \mathrm{~kg}(p=0.24)$ after 2 years (Fig. 1). TAT was also reduced after 9 months $(-3.0 \pm 0.8 \mathrm{~kg}$, $p=0.001)$ and after 2 years $(-2.8 \pm 1.2 \mathrm{~kg}, p=0.034)$. Accordingly, VAT and IHL were lowered at 9 months (VAT: $-0.46 \pm 0.12 \mathrm{~kg}, p=0.001$; IHL: $-3.7 \pm 1.9 \%$, $p=0.020$ ) and 2 years (VAT: $-0.50 \pm 0.17 \mathrm{~kg}, p=0.007$; IHL: $-1.8 \pm 0.6 \%, p=0.034)$ compared with baseline. Comparable changes were found in the database of the TULIP study and have been published elsewhere [3].

Cerebral insulin sensitivity as 'predictor' of changes in body weight, TAT, VAT and plasma glucose High baseline cerebral insulin sensitivity was significantly associated with
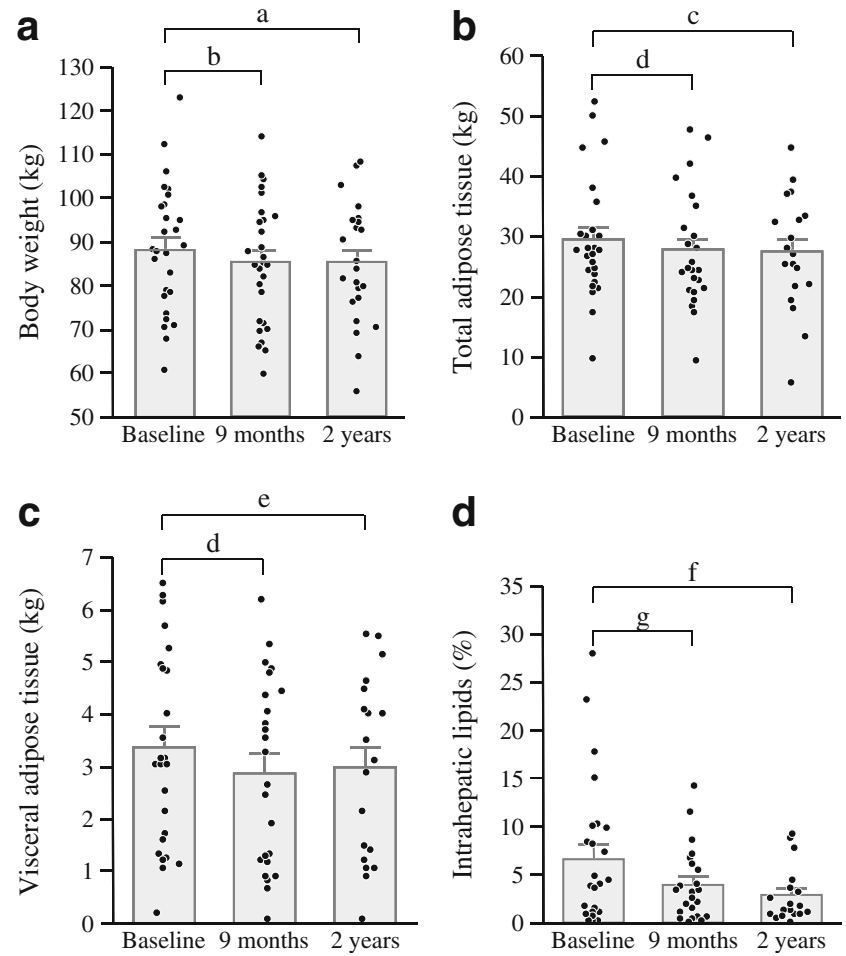

Fig. 1 Changes in body composition during the lifestyle intervention Body weight (a), total adipose tissue (b), visceral adipose tissue (c) and intrahepatic lipids (d) were reduced during the lifestyle intervention. ${ }^{\mathrm{a}} p=0.24,{ }^{\mathrm{b}} p=0.002,{ }^{\mathrm{c}} p=0.034,{ }^{\mathrm{d}} p=0.001,{ }^{\mathrm{e}} p=0.007,{ }^{\mathrm{f}} p=0.038$, $\mathrm{g}_{p}=0.020$

a more pronounced loss of TAT and VAT after 9 months and 2 years (Fig. 2 and Table 2). As measures for cerebral insulin sensitivity, we used the insulin-stimulated change in theta and beta activity. While TAT and VAT displayed significant results with both measures of cerebral insulin sensitivity, reduction in fasting plasma glucose was significantly associated with insulin-stimulated theta activity and only displayed a statistical trend for insulin-stimulated beta activity. However, loss of IHL and change in $2 \mathrm{~h}$ plasma glucose during the OGTT were not associated with cerebral insulin sensitivity measures independent of body weight change.

Cerebral insulin sensitivity and adherence to lifestyle recommendations During the lifestyle intervention, we assessed intake of food and specific nutrients by analysing food diaries and subsequent software-based determination of nutrients. Compared with baseline, mean energy intake was lower during the lifestyle intervention (baseline, 8,725 \pm $553 \mathrm{~kJ} /$ day; intervention, $7,423 \pm 368 \mathrm{~kJ} /$ day; $p=0.021$ ). Fat intake was reduced from $83 \pm 8 \mathrm{~g} /$ day to $64 \pm 3 \mathrm{~g}$ /day ( $p=0.009$ ). Accordingly, consumption of saturated fatty acids was reduced from $32 \pm 3 \mathrm{~g} /$ day to $24 \pm 1 \mathrm{~g} /$ day $(p=0.002)$. Relative to the energy intake, carbohydrate intake was increased (baseline, $45 \pm 2 \%$; intervention, $48 \pm 1 \%$; 

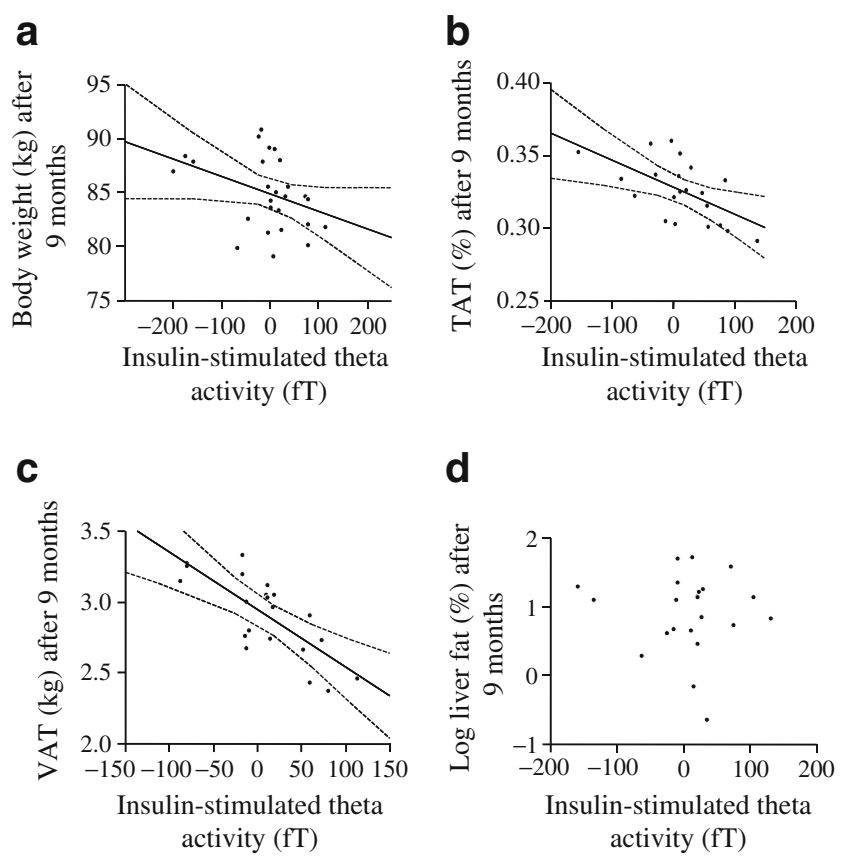

Fig. 2 High cerebral insulin sensitivity at baseline is associated with high loss in body fat. The change in body weight after 9 months of lifestyle intervention (a) displayed a statistical trend to a negative correlation with insulin-stimulated cerebrocortical theta activity at baseline $(r=-0.37, p=0.069)$. The changes in TAT $(r=-0.59$, $p=0.014)(\mathbf{b})$ and VAT $(r=-0.76, p=0.001)$ (c) correlated negatively with insulin-stimulated cerebrocortical theta activity at baseline. However, liver fat change (d) did not correlate with insulinstimulated cerebrocortical theta activity at baseline $(p=0.81)$. Body weight values were adjusted for baseline. TAT, VAT and liver fat values were adjusted for baseline, age, sex and body weight change

$p=0.028)$. There was no change in protein consumption (baseline, $16.3 \pm 0.6 \%$; intervention, $16.4 \pm 0.4 \%$; $p=0.79$ ).
Whereas there was no significant correlation between reduction in energy intake and insulin-stimulated theta and beta activity ( $p=0.61$ and $p=0.39$, respectively), the intake of macronutrients was associated with insulin-stimulated theta activity. In a multiple regression analysis correcting for sex and age, changes in the intake of fat $(r=-0.49$, $p=0.034)$ and saturated fatty acids $(r=-0.48, p=0.032)$ correlated negatively with insulin-stimulated theta activity (Figs 3a, b). Accordingly, there was a relative increase in carbohydrate intake, as suggested by a statistical trend toward a positive correlation between insulin-stimulated theta activity and carbohydrate consumption in percentage of total energy intake ( $r=0.39, p=0.087$ ) (Fig. 3c).

Retrospectively, participants who displayed high adherence to the lifestyle recommendations as expressed by reaching at least three of the intervention targets (fat intake $<30 \%$ of energy, saturated fatty acids $<10 \%$ of energy, fibre intake $>15 \mathrm{~g}$ per $4,187 \mathrm{~kJ}[1,000 \mathrm{kcal}]$, more than $3 \mathrm{~h}$ physical activity) were characterised by higher insulin-stimulated theta activity at baseline. This effect was mainly based on higher insulin-stimulated theta activity in participants reaching the target of physical activity (Fig. 4).

\section{Discussion}

Here we show that cerebral insulin sensitivity displayed a positive association with loss of total body fat (TAT) and specific fat depots. Individuals with high cerebral insulin sensitivity displayed more loss of TAT and VAT during the lifestyle intervention than those who were insulin resistant, as expressed by low insulin-stimulated beta and
Table 2 Correlations of changes in body composition and metabolism during lifestyle intervention with cerebral insulin sensitivity

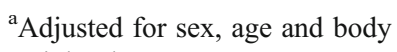
weight change

FPG, fasting plasma glucose; $2 \mathrm{~h}$ PG (OGTT), plasma glucose $2 \mathrm{~h}$ after ingestion of $75 \mathrm{~g}$ dextrose in the OGTT
}

\begin{tabular}{|c|c|c|c|c|}
\hline \multirow[t]{3}{*}{ Change } & \multicolumn{4}{|c|}{ Insulin-induced stimulation of: } \\
\hline & \multicolumn{2}{|c|}{ Theta activity } & \multicolumn{2}{|c|}{ Beta activity } \\
\hline & $r$ & $p$ value & $r$ & $p$ value \\
\hline Body weight (kg) 9 months & -0.37 & 0.069 & - & 0.18 \\
\hline Body weight $(\mathrm{kg}) 2$ years & - & 0.25 & - & 0.92 \\
\hline TAT (\%) 9 months $^{\mathrm{a}}$ & -0.59 & 0.014 & -0.61 & 0.021 \\
\hline TAT (\%) 2 years $^{\mathrm{a}}$ & -0.70 & 0.008 & -0.67 & 0.036 \\
\hline VAT (kg) 9 months $^{\mathrm{a}}$ & -0.76 & 0.001 & -0.64 & 0.013 \\
\hline VAT (kg) 2 years $^{\mathrm{a}}$ & -0.62 & 0.031 & -0.59 & 0.055 \\
\hline IHL (\%) 9 months $^{\mathrm{a}}$ & - & 0.63 & - & 0.16 \\
\hline IHL (\%) 2 years $^{\mathrm{a}}$ & - & 0.47 & - & 0.11 \\
\hline FPG (mmol/l) 9 months $^{\mathrm{a}}$ & -0.51 & 0.016 & -0.40 & 0.079 \\
\hline FPG $(\mathrm{mmol} / \mathrm{l}) 2$ years $^{\mathrm{a}}$ & -0.44 & 0.092 & - & 0.14 \\
\hline 2 h PG (OGTT, mmol/l) 9 months $^{\mathrm{a}}$ & - & 0.38 & - & 0.48 \\
\hline 2 h PG (OGTT, mmol/l) 2 years $^{\mathrm{a}}$ & - & 0.51 & - & 0.49 \\
\hline
\end{tabular}



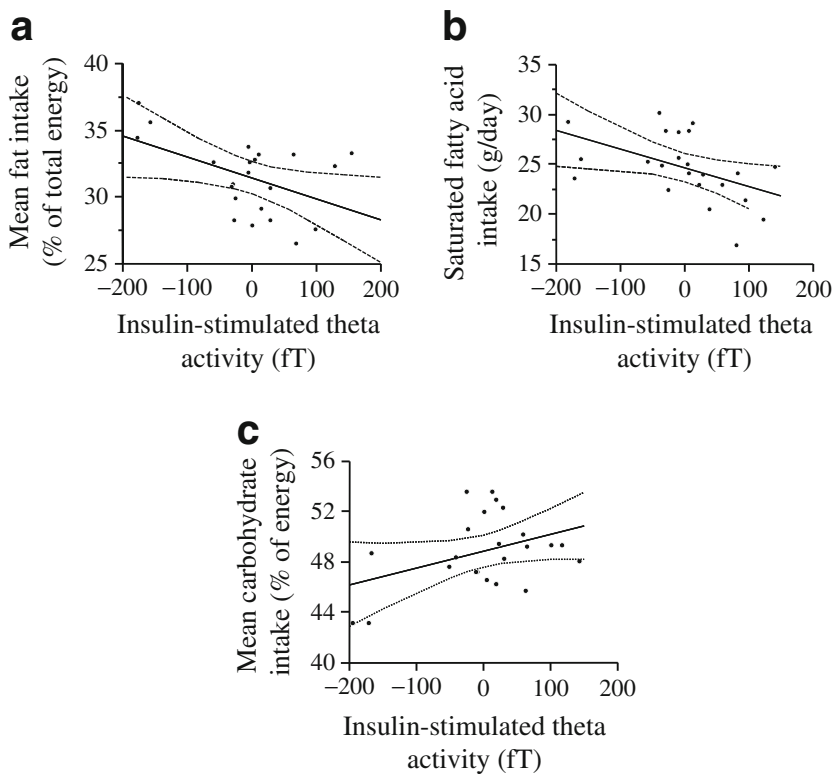

Fig. 3 Cerebral insulin sensitivity and adherence to diet recommendations. The decrease in fat intake $(r=-0.49, p=0.034)$ (a) and consumption of saturated fatty acids $(r=-0.48, p=0.032)$ (b) correlated negatively with insulin-stimulated cerebrocortical theta activity at baseline. However, the change in carbohydrate uptake (relative to total energy intake) showed a trend toward a positive correlation with insulin-stimulated cerebrocortical theta activity at baseline $(r=0.39, p=0.087)$ (c). The mean intakes of carbohydrate, saturated fatty acids and fat were adjusted for baseline, sex and age

theta activity. Lifestyle modification was induced by recurrent dietary counselling throughout the study. The individuals' adherence to the lifestyle recommendations was monitored by the dietitian. Evaluation of diet and activity protocols revealed that high baseline insulinstimulated theta activity was associated with a more pronounced decrease in fat intake, a relative increase in carbohydrate intake, and better compliance with the physical activity target.

In the brain, insulin is a known regulator of energy homoeostasis [4]. In animal studies, knock-out of the insulin receptor [10] and diet-induced deterioration of insulin signalling [11] led to obesity. Although most animal studies showing a direct link between insulin and control of body weight focused on hypothalamic insulin receptors, there is also evidence that cerebrocortical activity is modulated by insulin in parallel to changes in spontaneous locomotion [12]. In addition, induction of cerebral insulin resistance by a high-fat diet abolished these insulin effects on cortical activity and locomotion in these animals.

In humans, nasal administration of insulin can be used to specifically stimulate the brain. In a study with twice daily nasal insulin use, body weight and body fat were reduced after 8 weeks [13]. Interestingly, obese humans display insulin resistance of the cerebral cortex [5]. However, it is unclear so far whether insulin resistance of the cerebral
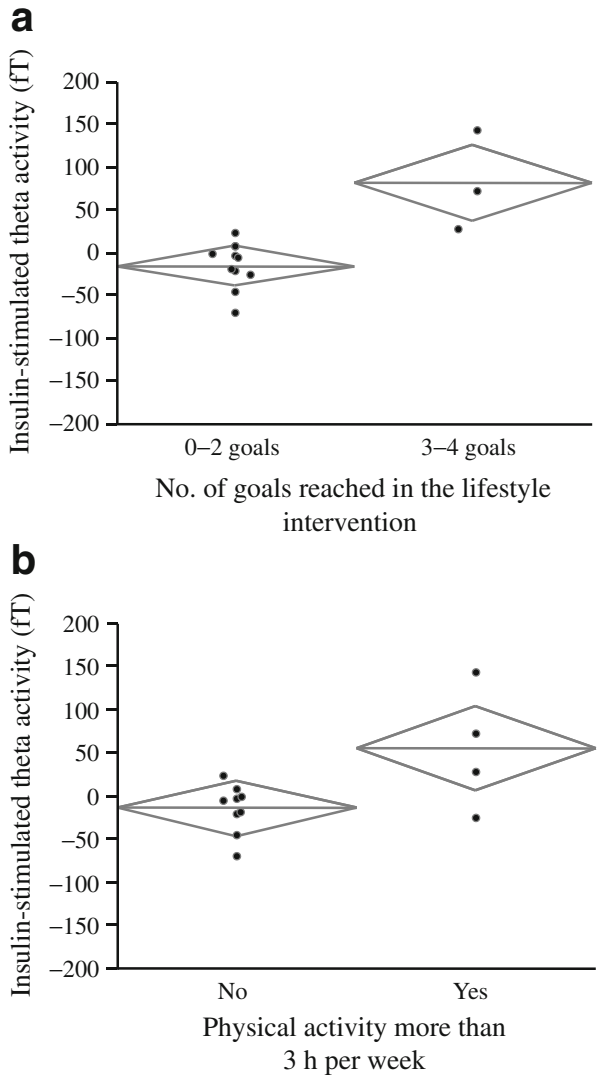

Fig. 4 Cerebral insulin sensitivity is associated with physical activity during lifestyle intervention. Insulin-stimulated cerebrocortical theta activity was higher at baseline in participants who reached three or more goals of the recommended lifestyle modifications $(p=0.010)$ (a) and especially in those participants who increased their physical activity to over $3 \mathrm{~h}$ per week $(p=0.037)(\mathbf{b})$

cortex in humans is a cause or consequence of obesity. To our knowledge, this study is the first to show that the cerebrocortical response to insulin is relevant for future development of body fat in humans.

In general, decreases in body fat and body weight can be explained by a shift of energy homoeostasis towards a relative deficit in energy intake by (1) reducing food intake, (2) increasing energy expenditure or (3) doing both. To further differentiate whether the insulin effect of cortical activity predicts reduction in food intake or increase in energy expenditure, we analysed the food and exercise diaries of our participants and scored the adherence to lifestyle recommendations.

High insulin sensitivity of the brain at baseline was especially present in participants who reached three or more goals of the lifestyle intervention. The strongest single goal was increase in physical activity. Those participants who spent more than $3 \mathrm{~h}$ per week in physical activity were characterised by higher insulin-stimulated theta activity at baseline than participants training less than $3 \mathrm{~h}$. Therefore, we speculate that insulin-stimulated cerebrocortical activity, 
especially in the theta band, may interfere with physical activity during a lifestyle intervention that causes increased energy expenditure and subsequent loss of body fat. This is well in line with a recent study in mice showing an increase in locomotor activity after intracerebroventricular injection of insulin accompanied by increases in cortical theta and delta activity [12].

High insulin sensitivity of the brain at baseline was associated with lower intake of fat, especially saturated fat, during the lifestyle intervention. Therefore high insulinstimulated theta activity may reflect high ability to follow dietary recommendations. However, it seems that these effects are not sufficient to induce reduction of total energy intake. Taking the results together, it is most likely that both reducing food, especially fat, intake and increasing energy expenditure are mechanisms that link high insulin sensitivity of the brain to decreases in body fat and body weight.

Recently, we observed that serum concentrations of saturated fatty acids correlated negatively with insulinstimulated theta activity [6]. At that time, we speculated that fatty acids derived from visceral fat depots may cause insulin resistance of the brain and therefore lead to further weight gain. Indeed, recent studies confirmed that intracerebroventricular palmitic acid infusion and high-fat diet induce insulin resistance in different brain regions in mice and rats $[12,14,15]$. These observations, together with the present finding of high insulin sensitivity in the brain associated with lower intake of saturated fat, may suggest that insulin resistance in the brain elevates serum concentrations of saturated fatty acids not only by increasing visceral fat mass, but also more directly by decreasing physical activity and increasing intake of saturated fat. This mechanism may contribute to maintenance of cerebral insulin resistance after it has been induced by a high-fat diet.

Although a number of our previous findings support that insulin-induced modulation of theta and beta activity represent functions related to body weight regulation, it should be noted that the raw values of these activities have low specificity. Beta activity is generated by the cerebral cortex and is characteristically involved in attention and multiple cognitive processes [16]. So far it is unclear whether beta activity interferes with cognitive control of eating behaviour. Theta activity, however, is mainly controlled by the hippocampus $[17,18]$, a region that plays a role in memory but also food reward mechanisms [19]. Furthermore, reduction in theta activity has been associated with the loss of locomotor activity and voluntary movement in rodents [20].

The major limitation of our study is that the number of participants undergoing measurement of cerebral insulin sensitivity is low. This means that every finding bears the possibility of false-positive results in the analyses. In addition, we can only present correlational data, although they are obtained in a prospective study using an established measurement of cerebrocortical activity during a hyperinsulinaemic-euglycaemic clamp and a placebo experiment.

In conclusion, our data suggest that insulin sensitivity of the brain predicts the individual reduction of weight and body fat during lifestyle interventions. In general, high insulin sensitivity of the brain was associated with stricter adherence to the recommended lifestyle modification, including increased physical activity as well as reduction of dietary fat and saturated fatty acids.

Acknowledgements We gratefully acknowledge the excellent technical assistance of A. Teigeler, E. Kollmar, M. Borutta and G. Walker. This study was supported by the Deutsche Forschungsgemeinschaft (KFO 114 and FR 1561/4-1) and the German Federal Ministry of Education and Research in the form of grants to the German Centre for Diabetes Research (DZD e.V.) and to the Kompetenznetzwerk Adipositas 01GI0849.

Contribution statement OT was responsible for the conception and design, analysis and interpretation of data and writing the article, and gave final approval of the version to be published. HP was responsible for conception and design, analysis and interpretation of data and revising the article, and gave final approval of the version to be published. AMH, TS, KTS and HUH were responsible for conception and design and revising the article, and gave final approval of the version to be published. MH, CK, NS, JM and ES were responsible for analysis and interpretation of data and revising the article, and gave final approval of the version to be published. AF was responsible for conception and design, analysis and interpretation of data and revising the article, and gave final approval of the version to be published.

Duality of interest The authors declare that there is no duality of interest associated with this manuscript.

\section{References}

1. Tuomilehto J, Lindstrom J, Eriksson JG et al (2001) Prevention of type 2 diabetes mellitus by changes in lifestyle among subjects with impaired glucose tolerance. N Engl J Med 344:1343-1350

2. Knowler WC, Barrett-Connor E, Fowler SE et al (2002) Reduction in the incidence of type 2 diabetes with lifestyle intervention or metformin. N Engl J Med 346:393-403

3. Thamer C, Machann J, Stefan N et al (2007) High visceral fat mass and high liver fat are associated with resistance to lifestyle intervention. Obesity 15:531-538

4. Schwartz MW, Woods SC, Porte D Jr, Seeley RJ, Baskin DG (2000) Central nervous system control of food intake. Nature 404:661-671

5. Tschritter O, Preissl H, Hennige AM et al (2006) The cerebrocortical response to hyperinsulinemia is reduced in overweight humans: a magnetoencephalographic study. Proc Natl Acad Sci USA 103:12103-12108

6. Tschritter O, Preissl H, Hennige AM et al (2009) The insulin effect on cerebrocortical theta activity is associated with serum concentrations of saturated nonesterified fatty acids. J Clin Endocrinol Metab 94:4600-4607

7. Matsuda A, DeFronzo R (1999) Insulin sensitivity indices obtained from oral glucose tolerance testing. Diabetes Care 22:1462-1470 
8. Machann J, Thamer C, Schnoedt B et al (2005) Standardized assessment of whole body adipose tissue topography by MRI. J Magn Reson Imaging 21:455-462

9. Schafer S, Kantartzis K, Machann J et al (2007) Lifestyle intervention in individuals with normal versus impaired glucose tolerance. Eur J Clin Invest 37:535-543

10. Bruning JC, Gautam D, Burks DJ et al (2000) Role of brain insulin receptor in control of body weight and reproduction. Science 289:2122-2125

11. Kaiyala KJ, Prigeon RL, Kahn SE, Woods SC, Schwartz MW (2000) Obesity induced by a high-fat diet is associated with reduced brain insulin transport in dogs. Diabetes 49:1525-1533

12. Hennige AM, Sartorius T, Lutz SZ et al (2009) Insulin-mediated cortical activity in the slow frequency range is diminished in obese mice and promotes physical inactivity. Diabetologia 52:2416-2424

13. Hallschmid M, Benedict C, Schultes B, Fehm HL, Born J, Kern W (2004) Intranasal insulin reduces body fat in men but not in women. Diabetes 53:3024-3029

14. Benoit SC, Kemp CJ, Elias CF et al (2009) Palmitic acid mediates hypothalamic insulin resistance by altering PKC-theta subcellular localization in rodents. J Clin Invest 119:2577-2589
15. Boghossian S, Lemmon K, Park M, York DA (2009) High-fat diets induce a rapid loss of the insulin anorectic response in the amygdala. Am J Physiol Regul Integr Comp Physiol 297:R1302R1311

16. Laufs H, Krakow K, Sterzer P et al (2003) Electroencephalographic signatures of attentional and cognitive default modes in spontaneous brain activity fluctuations at rest. Proc Natl Acad Sci USA 100:11053-11058

17. Tesche CD, Karhu J (2000) Theta oscillations index human hippocampal activation during a working memory task. Proc Natl Acad Sci USA 97:919-924

18. Stoeckel LE, Weller RE, Cook EW III, Twieg DB, Knowlton RC, Cox JE (2008) Widespread reward-system activation in obese women in response to pictures of high-calorie foods. NeuroImage 41:636-647

19. Pelchat ML, Johnson A, Chan R, Valdez J, Ragland JD (2004) Images of desire: food-craving activation during fMRI. NeuroImage 23:1486-1493

20. Dzoljic E, van Leeuwen R, de Vries R, Dzoljic MR (1997) Vigilance and EEG power in rats: effects of potent inhibitors of the neuronal nitric oxide synthase. Naunyn Schmiedebergs Arch Pharmacol 356:56-61 